\title{
Identification of Novel Mutation in EIF2AK3 Gene as a Causal Variant in a Family With Rare Disease
}

\author{
Raziyeh Khalesia ${ }^{\mathrm{a}}$, Masoud Garshasbia, b
}

\begin{abstract}
Wolcott-Rallison syndrome (WRS) is now identified as the most frequent cause of neonatal/early onset diabetes in patients that initiated before 6 months of age. Inheritance pattern of disease is autosomal recessive and most of patients are from populations with frequent consanguineous marriages. WRS is caused by mutations in $E I F 2 A K 3$ gene which encodes eukaryotic translation initiation factor $2 \mathrm{a}$ kinase. Several mutations have been reported in this gene and they are almost non-sense, frameshift, missense, and splice site. Here, we analyzed the $E I F 2 A K 3$ gene in an Iranian girl suspicious for WRS. Sequencing analysis of the gene identified a homozygous novel non-sense mutation in exon 9 of the gene, Y479X.
\end{abstract}

Keywords: WRS; EIF2AK3 gene; Novel mutation; Neonatal diabetes

\section{Introduction}

Wolcott-Rallison syndrome (WRS) (OMIM no. 226980) for the first time was recognized in three affected siblings by Drs Wolcott and Rallison [1]. This is now identified as the most frequent cause of neonatal/early onset diabetes in patients [2]. There are variable manifestations involved in WRS such as multiple epiphyseal dysplasia, growth retardation, osteopenia, mental retardation or developmental delay, hepatic and renal dysfunction [3,4]. Typically, diabetes initiates before 6 months of age, and skeletal dysplasia is recognized within 1 or 2 years of life. Other manifestations are different between patients based on their nature and severity [2].

WRS is known as a rare autosomal recessive disorder and most of affected individuals are from populations with frequent consanguineous marriages, such as the Middle-East,

Manuscript accepted for publication August 04, 2016

aDepartment of Medical Genetics, Faculty of Medical Sciences, Tarbiat Modares University, Tehran, Iran

${ }^{b}$ Corresponding Author: Masoud Garshasbi, Department of Medical Genetics, Faculty of Medical Sciences, Tarbiat Modares University, Tehran, Iran.

Email: masoud.garshasbi@modares.ac.ir

doi: https://doi.org/10.14740/jmc2597e
North Africa, Pakistan and Turkey [5].

WRS is caused by mutations in EIF2AK3 gene which encodes eukaryotic translation initiation factor 2 a kinase 3 , also known as pancreatic EIF2a kinase (PEK) and PKR-like endoplasmic reticulum kinase (PERK) [6]. PERK is a transmembrane protein located in the endoplasmic reticulum (ER), which plays a key role in the translation control during the unfolded protein response (UPR) [2]. So far, several mutations have been reported in this gene which led to the onset of WRS $[5,7,8]$. These mutations are non-sense, frameshift, missense, and splice site mutations which have been observed in both homozygous and compound heterozygous state [4, 8-15]. Here we report a novel non-sense mutation in $E I F 2 A K 3$ gene in an Iranian girl. These novel mutations can help in detection of other cases suspicious for WRS.

\section{Case Report}

A 12-year-old Iranian patient girl, born as a result of a consanguineous marriage, was referred to our lab. In medical history of the patient, it was noted that biochemical tests have been performed at 40 days after birth which had indicated neutropenia and high level of blood glucose (glucose $>500$ ). Therefore, insulin therapy had been applied. Since she was 4 years old, she had to use medical brace due to laxity of knee cartilage and dysgenesis of femoral head cartilages. She is not able to walk now and suffers from severe pain in pelvic area or femoral heads. Bone mineral density at her 8 years had been found to be below the expected range of age which has been improved by treatment.

Although she had received growth hormone for 7 months, it did not have positive effect on her short stature. Considering this medical history, the diagnosis of WRS was made based on clinical manifestations including pancreatic dysfunction, developmental delay, epiphyseal dysplasia and neutropenia.

Appropriate informed consent was obtained for all participants in the study, and institutional research ethics approval was obtained. The $10 \mathrm{~mL}$ peripheral bloods were collected from affected child and her parents in EDTA tubes. Genomic DNA was extracted from leukocytes using salting out standard method. The entire coding region and the exon-intron boundaries of the EIF $2 A K 3$ gene were amplified by PCR (primers and conditions are available upon request). All amplicons were sequenced by Sanger method (Applied Biosystems). Sequences 
were compared with the $E I F 2 A K 3$ reference sequence (accession no. AF110146.1) and any variant change found in the sequences was checked against published polymorphisms and for conservation across species.

Sequencing analysis of patient's $E I F 2 A K 3$ gene has identified a homozygous $T$ to $G$ substitution $(c .1437 T>G)$ in exon 9 of the gene. This exchange leads to a novel non-sense p.Tyr479Ter (p.Y479*) mutation in regulatory domain of protein. This mutation has not been reported so far in any of the publicly available databases for normal variants as well as 1000 genome project, EVS and HGMD. Subsequently, further molecular testing was performed in order to confirm the carrier status of her parents for this mutation and turned out that both parents are indeed heterozygous carriers for the p.Y479* mutation.

\section{Discussion}

WRS is a very scarce and challenging disease [16]. Less than 100 cases have been announced to date and most patients are from consanguineous families. Therefore, prevalence may vary notably between countries. Although WRS is a rare syndrome, it seems to be the most frequent form of neonatal/early onset permanent neonatal diabetes mellitus (PNDM) in patients born to consanguineous marriages, where it may be responsible for $25 \%$ of cases [5].

WRS should be discriminated from other forms of neonatal/early onset insulin-dependent diabetes based on clinical presentation and genetic testing. It is always permanent after disease onset, which simply distinguishes it from temporary neonatal diabetes caused by other genetic deficiencies $[17,18]$. Although WRS is easily distinguished from the other forms of PNDM based on clinical features, they may be misdiagnosed at the early stages, as all the clinical features of the syndrome are not yet expressed [2].

Rare autosomal recessive types of PNDM, which are related to several organ abnormalities, have been genetically characterized and are undoubtedly differentiated from WRS.

Screening for WRS should be doubted in any newborn who exhibits PNDM (before 6 months) or at a very young age specially if he/she is from a population with high rate of consanguinity, or in cases with history of neonatal diabetes with quickly fatal result in siblings or in the extended family; these patients should be evaluated for other clinical manifestations such as skeletal abnormality, growth retardation and short stature [2].

Although EIF2AK3 mutation screening verifies (or excludes) the WRS diagnosis [2], in practice genetic testing for this condition is usually delayed until the full clinical picture is evident and a clinical diagnosis of WRS is made [7].

Iran is one of the countries located in the consanguineous marriage belt; therefore the rate of recessive mutations (including $E I F 2 A K 3$ ) is expected to be higher in Iranian population compared to other populations [5]. The high frequency and spectrum of WRS has been already shown in other populations with high rate of consanguinity such as Saudi Arabia [19]. So far only one missense mutation (Q166R) has been reported in
Iranian patients [20].

Here we report an additional novel homozygous non-sense mutation (Y479X) in exon 9 of the EIF2AK3 gene, resulting in the protein truncation and leading to WRS. This has been corroborated by several recent reports of non-sense mutations found in different exons of the EIF $2 A K 3$ gene leading to protein truncation, and therefore WRS $[10,13,15]$.

Screening for $E I F 2 A K 3$ gene mutations can be recommended especially because WRS is now recognized as the most frequent cause of neonatal diabetes in children born to consanguineous marriages. Considering the recessive mode of inheritance, genetic screening becomes important as it can enable us to aid in risk prediction and clinical management. Of course availability of genetic testing facilities for clinicians will be also very important in order to confirm the diagnosis and provide families with proper genetic counseling.

\section{Acknowledgments}

We wish to thank all the members of this family for their willing participation and cooperation with this study.

\section{Funding Support}

This research was supported by a funding from the Wellcome Trust to Professors Andrew Hattersley and Sian Ellard at the University of Exeter Medical School (UK) for the Genetic testing of neonatal diabetes.

\section{Conflicts of Interest}

There are no actual or potential conflicts of interest in relation to this article.

\section{References}

1. Wolcott CD, Rallison ML. Infancy-onset diabetes mellitus and multiple epiphyseal dysplasia. J Pediatr. 1972;80(2):292-297.

2. Julier C, Nicolino M. Wolcott-Rallison syndrome. Orphanet J Rare Dis. 2010;5:29.

3. Sovik O, Njolstad PR, Jellum E, Molven A. WolcottRallison syndrome with 3-hydroxydicarboxylic aciduria and lethal outcome. J Inherit Metab Dis. 2008;31(Suppl 2):S293-297.

4. Durocher F, Faure R, Labrie Y, Pelletier L, Bouchard I, Laframboise R. A novel mutation in the EIF2AK3 gene with variable expressivity in two patients with WolcottRallison syndrome. Clin Genet. 2006;70(1):34-38.

5. Rubio-Cabezas O, Patch AM, Minton JA, Flanagan SE, Edghill EL, Hussain K, Balafrej A, et al. Wolcott-Rallison syndrome is the most common genetic cause of permanent neonatal diabetes in consanguineous families. J Clin Endocrinol Metab. 2009;94(11):4162-4170. 
6. Delepine M, Nicolino M, Barrett T, Golamaully M, Lathrop GM, Julier C. EIF2AK3, encoding translation initiation factor 2-alpha kinase 3, is mutated in patients with Wolcott-Rallison syndrome. Nat Genet. 2000;25(4):406409.

7. Senee V, Vattem KM, Delepine M, Rainbow LA, Haton C, Lecoq A, Shaw NJ, et al. Wolcott-Rallison Syndrome: clinical, genetic, and functional study of EIF2AK3 mutations and suggestion of genetic heterogeneity. Diabetes. 2004;53(7):1876-1883.

8. Ozbek MN, Senee V, Aydemir S, Kotan LD, Mungan NO, Yuksel B, Julier C, et al. Wolcott-Rallison syndrome due to the same mutation (W522X) in EIF2AK3 in two unrelated families and review of the literature. Pediatr Diabetes. 2010;11(4):279-285.

9. Iyer S, Korada M, Rainbow L, Kirk J, Brown RM, Shaw N, Barrett TG. Wolcott-Rallison syndrome: a clinical and genetic study of three children, novel mutation in EIF2AK3 and a review of the literature. Acta Paediatr. 2004;93(9):1195-1201.

10. Reis AF, Kannengiesser C, Jennane F, Manna TD, Cheurfa N, Oudin C, Savoldelli RD, et al. Two novel mutations in the EIF2AK3 gene in children with Wolcott-Rallison syndrome. Pediatr Diabetes. 2011;12(3 Pt 1):187-191.

11. Jahnavi S, Poovazhagi V, Kanthimathi S, Gayathri V, Mohan V, Radha V. EIF2AK3 mutations in South Indian children with permanent neonatal diabetes mellitus associated with Wolcott-Rallison syndrome. Pediatr Diabetes. 2014;15(4):313-318.

12. Triantafyllou P, Vargiami E, Vagianou I, Badouraki M, Julier C, Zafeiriou DI. Early-onset diabetes mellitus and neurodevelopmental retardation: the first Greek case of Wolcott-Rallison syndrome. J Pediatr Endocrinol Metab.
2014;27(9-10):967-970

13. Sang Y, Liu M, Yang W, Yan J, Chengzhu, Ni G. A novel EIF2AK3 mutation leading to Wolcott-Rallison syndrome in a Chinese child. J Pediatr Endocrinol Metab. 2011;24(3-4):181-184.

14. Al-Shawi M, Al Mutair A, Ellard S, Habeb AM. Variable phenotype in five patients with Wolcott-Rallison syndrome due to the same EIF2AK3 (c.1259delA) mutation. J Pediatr Endocrinol Metab. 2013;26(7-8):757-760.

15. Feng DR, Meng Y, Zhao SM, Shi HP, Wang WC, Huang SZ. [Two novel EIF2AK3 mutations in a Chinese boy with Wolcott-Rallison syndrome]. Zhonghua Er Ke Za Zhi. 2011;49(4):301-305.

16. Bin-Abbas B, Shabib S, Hainau B, Al-Ashwal A. WolcottRallison syndrome: clinical, radiological and histological findings in a Saudi child. Ann Saudi Med. 2001;21(12):73-74

17. Iafusco D, Stazi MA, Cotichini R, Cotellessa M, Martinucci ME, Mazzella M, Cherubini V, et al. Permanent diabetes mellitus in the first year of life. Diabetologia. 2002;45(6):798-804.

18. Edghill EL, Dix RJ, Flanagan SE, Bingley PJ, Hattersley AT, Ellard S, Gillespie KM. HLA genotyping supports a nonautoimmune etiology in patients diagnosed with diabetes under the age of 6 months. Diabetes. 2006;55(6):1895-1898.

19. Habeb AM. Frequency and spectrum of Wolcott-Rallison syndrome in Saudi Arabia: a systematic review. Libyan J Med. 2013;8(1):21137.

20. Behnam B, Shakiba M, Ahani A, Razzaghy Azar M. Recurrent Hepatitis in Two Iranian Children: A Novel (Q166R) Mutation in EIF2AK3 Leading to Wolcott-Rallison Syndrome. Hepat Mon. 2013;13(6):e10124. 